\title{
Data Mining Models to Predict Patient's Readmission in Intensive Care Units
}

\author{
Pedro Braga ${ }^{1}$, Filipe Portela ${ }^{2}$, Manuel Filipe Santos ${ }^{2}$ and Fernando Rua ${ }^{3}$ \\ ${ }^{1}$ Information System Department, ${ }^{2}$ Algoritmi Research Centre, University of Minho, Guimarães, Portugal, \\ ${ }^{3}$ Serviço de Cuidados Intensivos, Centro Hospitalar do Porto, Portugal \\ pnevoabraga@gmail.com, \{cfp,mfs\}@dsi.uminho.pt,fernandorua.sci@hgsa.min-saude.pt
}

Keywords: Readmission, Intensive Care, INTCare, Decision Support in Intensive Care Medicine, Data Mining, SWIFT.

Abstract: Decision making is one of the most critical activities in Intensive Care Units (ICU). Moreover, it is extremely difficult for health professionals to interpret in real time all the available data. In order to improve the decision process, classification models have been developed to predict patient's readmission in ICU. Knowing the probability of readmission in advance will allow for a more efficient planning of discharge. Consequently, the use of these models results in a lower rates of readmission and a cost reduction, usually associated with premature discharges and unplanned readmissions. In this work was followed a numerical index, called Stability and Workload Index for Transfer (SWIFT). The data used to induce the classification models are from ICU of Centro Hospitalar do Porto, Portugal. The results obtained so far, in terms of accuracy, were very satisfactory $(98.91 \%)$. Those results were achieved through the use of Naïve Bayes technique. The models will allow health professionals to have a better perception on patient's future condition in the moment of the hospital discharge. Therefore it will be possible to know the probability of a patient being readmitted into the ICU.

\section{INTRODUCTION}

Ensuring that in a given situation are taken the better decisions, remains a huge challenge for science and technology. Thus, the Information Technology (IT) has been playing a crucial role in the adoption of measures and optimization of processes in organizations. The field of medicine is no exception, and the needs for rapid development and the use of IT as a response to various problems is increasing. As an example, there is intensive medicine, where critically ill patients need special care for their survival. The readmission of patients in Intensive Care Units (ICU) is currently one of the major problems and challenges of health professionals in the field of intensive care medicine, because it can be danger to the patient and spend extra resources. Since the ICU readmissions are common and costly, there is a need to investigate the application of new techniques to reduce unplanned readmission of patients into the ICU, because the currently existing techniques do not provide satisfactory results (Gortzis, Sakellaropoulos, Ilias, Stamoulis, \& Dimopoulou, 2008).

This study aims to demonstrate that by using data mining (DM) techniques it was possible to determine the probability of patient's readmission in order to assist health professionals in their decision process.

This article is structured in the following way: Introduction which is presented a brief introduction about the environment and the foundations of the work to develop. Background: where is presented the problem and some theoretical fundaments: Intensive Medicine, Readmissions, INTCare and the Stability and Index for Load Transfer. Study Description: describes the process of project development. Discussion of Results: are discussed and interpret the results obtained. Conclusions: where are presented the most important conclusions of the work. Future Work: presents recommendations for future work.

\section{BACKGROUND}

\subsection{Intensive Medicine}

Intensive Medicine (IM) is a medical specialty whose main goals are to diagnose and treat patients with serious illnesses and restore them to their previous state of health. IM can still be set up as a 
"Multidisciplinary field of medical science that specifically addresses three stages: prevention, diagnosis and treatment of patients with potentially reversible pathophysiological conditions that threaten or present failure of one or more vital functions" (Silva, 2007). Associated with IM comes the Intensive Care Units (ICU). ICUs are characterized as qualified locals to assume full responsibility for patients with organ dysfunction, supporting, preventing and reversing failure of vital organs (Ministério da Saúde, 2003). Intensivist is a health professional with critical care training that works in the ICU.

\subsection{ICU Readmission}

An unplanned readmission of patients is directly related to a bad decision by the intensivist at the time of patient assessment (discharge). However, the ability to predict relapse of a patient after the discharge from the ICU is limited (Gajic, et al., 2008). In order to understand how it is processed the readmission of a patient it is important first to realize how it is processed an admission. The admission into UCI is, by definition, "a time of transition for some patients whose life is at risk and it is part of a process and not an end in itself" (Ministério da Saúde, 2003). It is considered admission when the patient admitted to the health facility occupies a bed or couch for a minimum of 24 hours (ACSS, 2012). A patient is considered readmitted if he/she is hospitalized at the same hospital with the same principal diagnosis within thirty days after discharge (ACSS, 2012). According to literature review, in North America and Europe, the average rate of readmission of patients in ICUs is around 7\%. A study conducted by the Royal Melbourne Hospital in Australia showed that the rate of readmission of patients was $10.5 \%$. The main factors can be respiratory and cardiac problems, the progression of the patient's condition, care needs post-operative, and inadequate follow-up care (Russell, 1999). A study conducted in England by SSentif Intelligence (Intelligence, 2013), showed that on average $16 \%$ of patients above 75 years of age suffer readmission 28 days after discharge, although this figure varies significantly across the country, in the West South England has an average of $12.98 \%$ and the city of London register a value of $17.06 \%$

\subsection{Stability and Workload Index for Transfer}

It is extremely difficult for the health professionals to interpret almost instantly all the data available. In fact, at the time of admission or discharge of the patient the criteria employed by the health professional are often subjective and are not likely to be reproduced in other cases. Many of these professionals are often forced to rely on their intuition and subjective analysis to assess the clinical status of the patient and thus determine whether the patient is ready for discharge or not (Gajic, et al., 2008).

Published data shows that there are models or mathematical techniques that help predict readmission of patients in the ICU. As an example, according to Gajic (2008), there is a study to develop and validate a numerical index called Stability and Workload Index for Transfer (SWIFT) (Gajic, et al., 2008). The considered variables to be used in SWIFT in order to estimate the probability of unplanned readmission were: length of stay in the ICU (duration in days), the source of the patient's admission, the Glasgow coma scale (GCS), the partial pressure of oxygen in arterial blood [PaO2] / and the fraction of inspired oxygen [FIO2] and evaluation of nursing care for respiratory problems [PCO2].

The final score is derived from a set of information available at the time of hospital discharge estimating the probability of the patient in the ICU using as support the scores presented in Table 1.

Table 1: SWIFT Variables and scores

\begin{tabular}{|c|c|}
\hline Variables & Score \\
\hline \multicolumn{2}{|l|}{ Original source of this ICU admission } \\
\hline Emergency department. & 0 \\
\hline Transfer from a ward or outside hospital & 8 \\
\hline \multicolumn{2}{|l|}{ Total ICU length of stay (duration in days) } \\
\hline$<2$ & 0 \\
\hline $2-10$ & 1 \\
\hline$>10$ & 14 \\
\hline \multicolumn{2}{|l|}{ Last measured $\mathrm{PaO} 2 / \mathrm{FIO} 2$ ratio } \\
\hline$>400$ & 0 \\
\hline$<400$ and $\geq 150$ & 5 \\
\hline$<150$ and $\geq 100$ & 10 \\
\hline$<100$ & 13 \\
\hline \multicolumn{2}{|l|}{ Glasgow Coma Scale at time of ICU discharge } \\
\hline$>14$ & 0 \\
\hline $11-14$ & 6 \\
\hline $8-10$ & 14 \\
\hline$<8$ & 24 \\
\hline \multicolumn{2}{|l|}{ Last arterial blood gas $\mathrm{PaCO} 2$} \\
\hline$<45 \mathrm{~mm} \mathrm{Hg}$ & 0 \\
\hline$>45 \mathrm{~mm} \mathrm{Hg}$ & 5 \\
\hline
\end{tabular}

SWIFT is according to some experts from ICU of Centro Hospitalar do Porto (CHP) the most popular readmission technique currently used in Portuguese hospitals. Therefore this predictive model was the basis of the current study using DM techniques. 


\subsection{INTCare}

This study is being developed under the research project called INTCare. INTCare is an Intelligent Decision Support System (IDSS) for Intensive Care Medicine, and is implemented in ICU of the Hospital de Santo António, CHP. The main objective was to change the responsiveness of reactive response to proactive, thus enabling physicians to ensure an improvement in the treatment of their patients (Portela, et al., 2012). The system uses a set of variables to try to predict the patient's condition through intelligent autonomous agents that are responsible for the fulfilment of objectives, which include the use of data mining to predict organ failure and the outcome of a patient in the next 24 hours. Currently this approach (predict readmissions) is part of one of the goals of INTCare II project recently approved by the Foundation for Science and Technology. INTCare System consists of four subsystems within which stand the system of data acquisition, the knowledge management, inference and the interface. For more information about the structure of INTCare it is possible to check on the following article (Portela, Pinto, \& Santos, 2012).

\section{STUDY DESCRIPTION}

\subsection{Methods and Tools}

For this study it was used the purpose of forecasting through a set of classification models. For the work were used Oracle SQL Developer for data treatment, $\mathrm{R}$ for data analysis and the Oracle Data Miner (ODM) to build prediction models (Taft, et al., 2003). Due the type of techniques available in ODM it was used: Support Vector Machines (SVM), Decision Trees (DT) and Naïve Bayes (NB)

\subsection{Business and Data Understanding}

The Business goal of this study is decreasing the readmissions number through the use of Data Mining. The Data Mining objective is developing models which allow determine the probability of patient readmission. These models act as a support to health professionals in decision-making and improving the patient's health services. To this study it was used real data provided from CHP databases. Although the data acquisition subsystem incorporate five different data sources (Portela, Pinto, \& Santos, 2012), the attributes used were collected in real time from only two different sources: Laboratory (LAB) and Clinical
Process (CP) - Electronic Health Record (EHR). Table 2 presents the attributes, type, domain and source of the information available.

Table 2: Attributes overview

\begin{tabular}{|c|c|c|c|}
\hline Attributes & Type & Domain & Source \\
\hline episode & number & - & LAB/CP \\
\hline nprocess & number & - & $\mathrm{LAB} / \mathrm{CP}$ \\
\hline nsample & varchar & - & $\mathrm{LAB}$ \\
\hline daten & date & - & $\mathrm{CP}$ \\
\hline sex & varchar & {$[\mathrm{M} ; \mathrm{F}]$} & $\mathrm{CP}$ \\
\hline po2 & number & {$[0 ;+\infty[$} & $\mathrm{LAB}$ \\
\hline fio2 & number & {$[0 ; 100]$} & $\mathrm{LAB}$ \\
\hline Pco2 & number & {$[0 ;+\infty[$} & $\mathrm{LAB}$ \\
\hline emergencyroom & varchar & {$[$ True;False $]$} & $\mathrm{CP}$ \\
\hline input date & date & - & $\mathrm{CP}$ \\
\hline output date & date & - & $\mathrm{CP}$ \\
\hline validation pco2 & date & - & $\mathrm{LAB}$ \\
\hline validation po2 & date & - & $\mathrm{LAB}$ \\
\hline
\end{tabular}

To this work it was evaluated a set of episodes with admission and discharges in the ICU, since December 20, 2010 to April 23, 2013. The attribute Glasgow coma scale (GCS) was not considered in this study because it is an attribute registered manually and consequently, countless null values were found.

\subsection{Data Preparation}

At this stage it was conducted an Extract Transforming and Loading (ETL) process, the number of times needed until they present quality. In this phase one of the main goal is to build new data from the existing data. The following derivations of new attributes were made in the dataset and can be seen in Figure 1.

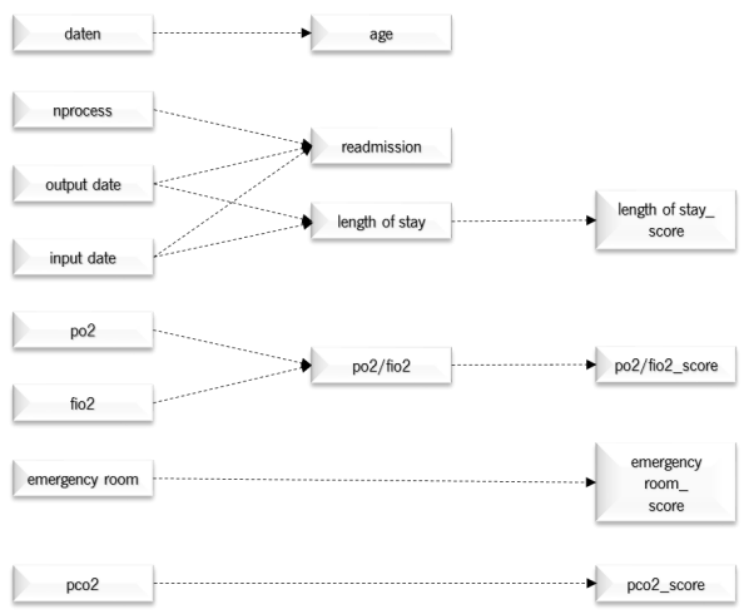

Figure 1: Schematic construction of new data 
The scores come from assigning values according to the score table SWIFT. Observing Table 1, and ignoring the GCS, variables in the analysis are: emergency room, length of stay, PO2/FIO2 and PCO2. Table 1 made a point between variables and their respective scores according to SWIFT. Table 1 also presented the scores domain.

New attributes:

- Age: Domain $[15 ;+\infty[$

- Readmission: Domain $[0,1]$

- Length of Stay: Domain $[1,76]$

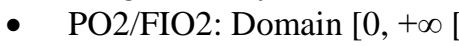

Scores:

- Emergency Room

- Length of Stay

- $\quad \mathrm{PO} 2 / \mathrm{FIO} 2$

- $\mathrm{PCO} 2$

In order to induce classification models some of the input attributes needed to be transformed. The point is to transform the attributes into discrete and normalized values in order to be used.

For the attributes PO2/FIO2, PCO2 and Length of Stay was necessary to convert the continuous numeric data into class intervals. For this purpose it was used an approach of equitable distribution of data classes. Attributes $\mathrm{PO} 2 / \mathrm{FIO} 2$ and $\mathrm{PCO} 2$ were equitably distributed however attribute Length of Stay had a slightly different approach.

The distribution followed the evaluation criteria of health professionals from CHP, and the attribute Length of Stay was divided into 3 parts: patients up to 1 day in hospital, in other words, sick passengers in ICU, patients between 2 and 5 days of hospitalization, so patients without extra complications, like infections or allergies, and patients with more than 5 days of admission, i.e., patients in serious condition with high probability of extra complications. In Table 3 it is possible to observe the division of classes.

Table 3: Data overview of the classes created by attribute

\begin{tabular}{|c|r|r|}
\hline Attribute & Class & $\%$ \\
\hline \multirow{4}{*}{ PO2/FIO2 } & {$[28-120[$} & 14.7 \\
\cline { 2 - 3 } & {$[120-167[$} & 14.0 \\
\cline { 2 - 3 } & {$[167-214[$} & 14.1 \\
\cline { 2 - 3 } & {$[214-265[$} & 14.3 \\
\cline { 2 - 3 } & {$[265-328[$} & 14.2 \\
\cline { 2 - 3 } & {$[328-430[$} & 14.2 \\
\hline \multirow{4}{*}{ PCO2 } & {$[430-39250[$} & 14.5 \\
\cline { 2 - 3 } & {$[11,3-32,5[$} & 14.0 \\
\hline
\end{tabular}

\begin{tabular}{|c|r|r|}
\hline \multirow{4}{*}{} & {$[35,9-38,8[$} & 13.9 \\
\cline { 2 - 3 } & {$[38,8-41,4[$} & 14.0 \\
\cline { 2 - 3 } & {$[41,4-44,7[$} & 14.3 \\
\cline { 2 - 3 } & {$[44,7-50[$} & 14.1 \\
\cline { 2 - 3 } Length of & {$[50-134]$} & 15.5 \\
\cline { 2 - 3 } Stay & {$[1]$} & 34.4 \\
\cline { 2 - 3 } & {$[2-5[$} & 37.8 \\
\hline \multirow{2}{*}{\begin{tabular}{c} 
Ly \\
\cline { 2 - 3 }
\end{tabular}} & {$[5-76]$} & 27.8 \\
\hline
\end{tabular}

\subsection{Modelling}

This phase focused on getting models to translate business goals through the application of data mining techniques. The process suffered two approaches, one for the normal table generated (score_swift_A) and one for a second table (score_swift_B) which suffered an oversampling. The necessity of using the oversampling technique is related to the existent dominance of a type of data distribution over the other. When facing this imbalance, the models tend to have a greater number of errors over the minority of the data distribution. So the oversampling through the data replication, increase the weight of minority data distribution, in order to be able to obtain a better performance of the classification models (Barua, Islam, \& Murase, 2013).

In order to understand what has been done in the $1^{\text {st }}$ approach (A) the balance is $98.66 \%$ for no readmitted patients and $1,34 \%$ for readmitted patients. For the $2^{\text {nd }}$ approach (B) the balance is $51 \%$ for no readmitted patients and $49 \%$ for readmitted patients. This takes into account two different approaches and eight scenarios using or not CaseMix.

CaseMix is generally represented as non-clinical data collected from the patient which may be useful for analysis and diagnosis or for research purposes.

For the design and evaluation of the models were used $70 \%$ of the data for training and the remaining $30 \%$ for the test. The training block is used to produce the model and the test is used to measure the ability of generalization of the model. The test block is also the percentage of data that are not used in the learning process. The encoding model is presented as follows:

$$
\begin{aligned}
\text { Target }(\text { dependent })= & \text { Readmission; } \\
\text { Input }(\text { independent })= & \{\text { Normal, Scores, Class and } \\
& \text { CaseMix }\} ;
\end{aligned}
$$

Normal $=\{$ pco 2, po $2 /$ fio 2, emergencyroom, length of stay $\}$

Scores $=\{$ pco2_score, po2/fio2_score, emergencyroom_score, lengthof stay_score 
Classes $=\{$ class_pco2, class_po2/fio2, class_lengthofstay, emergencyroom $\}$

CaseMix $=\{$ sex, age $\}$

Models applied to the two approaches are presented as follows:

$M 1=\{$ Normal + Scores $\}$

M2 $=\{$ Scores $\}$

M3 $=\{$ Normal $\}$

M4 $=\{$ Normal + Scores + Class $\}$

M5 $=\{$ Normal + Scores + CaseMix $\}$

M6 $=\{$ Scores + CaseMix $\}$

$M 7=\{$ Normal + CaseMix $\}$

$M 8=\{$ Normal + Scores + Class + CaseMix $\}$

Taking into account the two approaches it was developed a total of 48 models: 2 approaches $* 8$ scenarios $* 3$ techniques $* 1$ target.

In the construction of classification models the parameters were catalogued using oracle data mining according to the representation in Table 4.

Table 4 - Techniques Settings

\begin{tabular}{|c|c|}
\hline Description & Values \\
\hline Algorithm Name & Support Vector Machine \\
\hline Kernel Function & Gaussian \\
Tolerance Value & 0,001 \\
Active Learning & yes \\
\hline Algorithm Name & Decision Tree \\
\hline Homogeneity Metric & Gini \\
Maximum Depth & 7 \\
\hline Algorithm Name & Naive Bayes \\
\hline Pairwise Threshold & 0 \\
Singleton Threshold & 0 \\
\hline
\end{tabular}

\subsection{Evaluation}

The evaluation phase focuses primarily on the assessment and comparison of the results, noting whether they meet the initial goals of the project. All models were reviewed and evaluated in order to find influencing factors which may have escaped at earlier stages. In order to prove the results for the set of models developed for the readmission of patients, it is possible to observe in Tables 5 and 6 the attained results in terms of the three metrics: accuracy, sensitivity and specificity. In Table 5 there are the best three models in terms of accuracy. As it is possible observe the approach $\mathrm{B}$ is the one that presents the best results and the Naive Bayes figure as the technique that reaches the highest values of acuity most precisely with models NB_M7B and NB_M5B. Also in line with the results obtained, it is possible to observe through Table 6 the best 3 models for each metric and approach.

Table 5: Best models results.

\begin{tabular}{|c|c|c|c|c|}
\hline & & Accuracy & Sensitivity & Specificity \\
\hline $1^{\circ}$ & NB_M7B & $98.91 \%$ & $99.67 \%$ & $98.12 \%$ \\
\hline $2^{\text {o }}$ & NB_M5B & $98.12 \%$ & $98.02 \%$ & $98.23 \%$ \\
\hline $3^{\circ}$ & DT_M5B & $96.77 \%$ & $98.51 \%$ & $95.02 \%$ \\
\hline
\end{tabular}

Table 6: Best models for approach A and approach B

\begin{tabular}{|c|c|c|c|}
\hline A & Sensitivity & Specificity & Accuracy \\
\hline $1^{\circ}$ & $\begin{array}{c}\text { DT_M4A } \\
99.63 \%\end{array}$ & $\begin{array}{c}\text { SVM_M4A } \\
71.56 \%\end{array}$ & $\begin{array}{c}\text { NB_M5A } \\
94.07 \%\end{array}$ \\
\hline \multirow{2}{*}{$2^{\circ}$} & $\begin{array}{c}\text { DT_M1A } \\
99.52 \%\end{array}$ & $\begin{array}{c}\text { SVM_M2A } \\
47.48 \%\end{array}$ & $\begin{array}{c}\text { NB_M7A } \\
92.51 \%\end{array}$ \\
\hline \multirow{2}{*}{$3^{\circ}$} & $\begin{array}{c}\text { SVM_M8A } \\
99.52 \%\end{array}$ & $\begin{array}{c}\text { SVM_M1A } \\
\text { 42.92\% }\end{array}$ & $\begin{array}{c}\text { NB_M8A } \\
91.96 \%\end{array}$ \\
\hline B & Sensitivity & Specificity & Accuracy \\
\hline \multirow{2}{*}{$1^{\circ}$} & $\begin{array}{c}\text { NB_M7B } \\
99.67 \%\end{array}$ & $\begin{array}{c}\text { NB_M5B } \\
98.23 \%\end{array}$ & $\begin{array}{c}\text { NB_M7B } \\
98.91 \%\end{array}$ \\
\hline $2^{\circ}$ & $\begin{array}{c}\text { DT_M5B } \\
98.51 \%\end{array}$ & $\begin{array}{c}\text { NB_M7B } \\
98.12 \%\end{array}$ & $\begin{array}{c}\text { NB_M5B } \\
98.12 \%\end{array}$ \\
\hline $3^{\circ}$ & $\begin{array}{c}\text { DT_M7B } \\
98.51 \%\end{array}$ & $\begin{array}{c}\text { SVM_M7B } \\
95.77 \%\end{array}$ & $\begin{array}{c}\text { DT_M5B } \\
96.77 \%\end{array}$ \\
\hline
\end{tabular}

In order to evaluate the results and to compare the classification models for patients at the ICU readmission, an analysis was performed through the Receiver Operating Characteristic (ROC). The analysis via ROC curves, which were recently introduced in the field of DM (Prati, Batista, \& Monard, 2008) have become a powerful tool in the evaluation of classification models. Currently, the ROC curves are widely used in medicine, taking into account its extreme importance in the evaluation of medical treatments. A point near $100 \%$ is the perfect model, i.e., all positive and negative examples are correctly classified. From Figure 2 it was observed that the model NB_M7_CASEMIX_B is what is closest to the perfect spot, presenting itself as the best classification model.

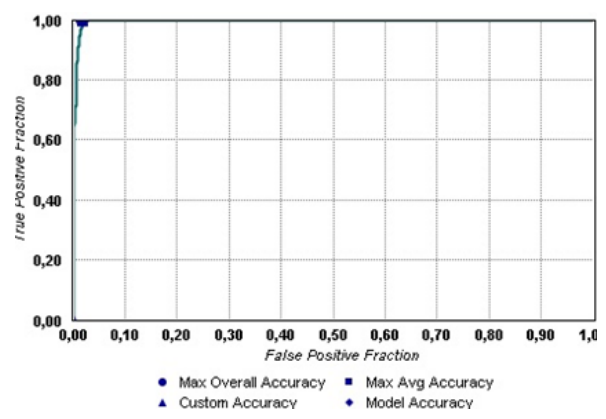

Figure 2: ROC for model NB_M7_CASEMIX_B 


\section{DISCUSSION OF RESULTS}

The best classification model in terms of accuracy is the NB_M7B with a precision of $98.91 \%$, followed by NB_M5B with $98.12 \%$ and DT_M5B with 96 , $77 \%$.

The results showed in table 6 corroborated the idea of being necessary the use of oversampling techniques. As can be observed for example to the models without oversampling the best specificity result it is $71.56 \%$ and for the models with oversampling it is $98.23 \%$. In fact before the use of the oversampling technique (A) the results were poor, however after the implementation of oversampling (B) the results improved significantly. One thing that is noticeable is that most of the top ranking models in this study are given through the technique of DM Naive Bayes. Based on the results obtained so far it is possible conclude that to obtain good results it was important to include other non-clinical attributes of the patient like age and sex.

\section{CONCLUSIONS}

This work contributed with a set of classification models that can help health professionals in the decision making. The models were introduced in order to predict whether a patient will be readmitted, or whether a patient won't be readmitted. The adoption of these models may result not only in reducing the high mortality associated to early bad discharges, but also can contribute to a reduction of costs of institutions. Furthermore, the impact of these models on the ICU will be huge, because allows the health professionals to use them as a decision support and, consequently, improve the health care of patients.

\section{FUTURE WORK}

Completed this study it is important to draw some prospective directions. Future work will include: To create new models through the exploration and inclusion of new attributes; To determine an evaluation metric of the models; To create patterns (clustering) for readmission through the generated models and integrate the developed models in a decision support system, in particular INTCare and optimize the developed models.

\section{ACKNOWLEDGEMENTS}

This work is supported by FEDER through Operational Program for Competitiveness Factors COMPETE and by national funds though FCT Fundação para a Ciência e Tecnologia in the scope of the project: FCOMP-01-0124-FEDER-022674.

The authors would like to thank FCT (Foundation of Science and Technology, Portugal) for the financial support through the contract PTDC/EIA/72819/ 2006 (INTCare) and PTDC/EEISII/1302/2012 (INTCare II).

\section{REFERENCES}

ACSS, 2012. Administração Central do Sistema de Saúde, Circular Normativa $n^{o} 33 / 2012$ Available at: http://www.acss.min-saude.pt

Barua, S., Islam, M. \& Murase, K., 2013. ProWSyn: Proximity Weighted Synthetic Oversampling Technique for Imbalanced Data Set Learning. Advances in Knowledge Discovery and Data Mining. s.1.:Springer Berlin Heidelberg, pp. 317-328.

Chapman, P. et al., 1999. CRISP-DM 1.0, s.1.: SPSS

Gajic, O. et al., 2008. The Stability and Workload Index for Transfer score predicts unplanned intensive care unit patient readmission: Initial development and validation. Crit Care Med. 676-682.

Gortzis, L. G. et al., 2008. Predicting ICU survival: A metalevel approach, s.l.: BMC Health Services Research.

Intelligence, S., 2013. Hospital Readmissions on the Increase, press release.

Ministério da Saúde, 2003. Cuidados Intensivos: Recomendações para o seu desenvolvimento.

Portela, F., Pinto, F. \& Santos, M. F., 2012. Data Mining Predictive Models for Pervasive Intelligent Decision Support in Intensive Care. Advances in Information Systems and Technologies.

Portela, et al., 2012. Intelligent and Real Time Data Acquisition and Evaluation to Determine Critical Events in Intensive Medicine. Procedia Technology.

Prati, Batista \& Monard, 2008. Curvas de ROC para avaliação de classificadores. pp. 215-222.

Russell, S., 1999. Reducing readmissions to the intensive care unit.. Heart Lung.

Santos, M. F., Mathew, W. \& Portela, C. F., 2011. Grid data mining for outcome prediction in intensive care medicine, s.l.: Springer CCIS.

Silva, A., 2007. Modelos de Inteligência Artificial na análise da monitorização de eventos clínicos adversos, Disfunção/Falência de órgãos e prognóstico do doente, Universidade do Porto, PHD Thesis;

Taft, M. et al., 2003. Oracle Data Mining Concepts, $10 \mathrm{~g}$ Release 1 (10.1), s.1.: Oracle Corporation. 